\title{
Changes in China's Food Self- Sufficiency Rate in the Context of a Changing Dietary Structure
}

\author{
Xiangzheng Deng, Institute of Geographic Sciences and Natural Resources Research, Chinese Academy of Sciences, \\ China* \\ Tianxiang Yue, Institute of Geographic Sciences and Natural Resources Research, Chinese Academy of Sciences, China \\ Yujie Liu, Institute of Geographic Sciences and Natural Resources Research, Chinese Academy of Sciences, China \\ Jingwei Dong, Institute of Geographic Sciences and Natural Resources Research, Chinese Academy of Sciences, China \\ Zhigang Sun, Institute of Geographic Sciences and Natural Resources Research, Chinese Academy of Sciences, China \\ Mingxing Chen, Institute of Geographic Sciences and Natural Resources Research, Chinese Academy of Sciences, China \\ Wenjiao Shi, Institute of Geographic Sciences and Natural Resources Research, Chinese Academy of Sciences, China \\ Xuezhen Zhang, Institute of Geographic Sciences and Natural Resources Research, Chinese Academy of Sciences, China \\ Zhe Zhao, Liaoning University, China \\ Ziyue Yu, The University of Nottingham, Ningbo, China \\ (iD) https://orcid.org/0000-0002-9760-4091
}

\begin{abstract}
This paper explores the impacts of the evolution of the dietary structure of the population on national food security using the food self-sufficiency rate under various statistical dimensions and simulate various food security scenarios for China in the medium- and long-term future. This study indicates that China's rapid economic and social development and the continuous improvement in people's living standards appears to be declining in the food self-sufficiency rate. The analysis regarding the factors influencing the food self-sufficiency rate revealed that the improved dietary structure, involving an increase in animal-based food product consumption, has led to a decrease in China's food selfsufficiency rate. It concludes that the dietary structure is likely to continue to move toward increased consumption of animal-based food products in China for the future. The research results will assist decision makers in formulating scientific food self-sufficiency rate targets and food security strategies.
\end{abstract}

\section{KEYWORDS}

Changing Dietary Structure, China, Dietary Structure, Food Self-Sufficiency

\section{INTRODUCTION}

China is promoting the strategy of rural revitalization, and it is significant to ensure national food security. Especially under the uncertain situation of the food market caused by the COVID-19, it is critical for rural revitalization to ensure adequate food and essential agricultural products. Some case areas in China, such as Yuanping City in Shanxi Province on the Loess Plateau, Qingyuan County 
in the Northeast Forest Region, and Kulun Banner in Inner Mongolia, are particularly noteworthy in food production capacity and its impact on regional self-sufficiency of food. Furthermore, the number of people facing severe food insecurity will surge globally due to the COVID-19, which means that people are at risk of death, malnutrition, or irreversible lifelong effects due to severe hunger. In addition, some food-exporting countries have taken measures to restrict exports to ensure their food supply in response to the potential economic stagnation and decline in production caused by the spread of the epidemic, raising concerns about the world's food supply. In this context, it is particularly urgent and essential to analyze the changing dietary structure and its impact on China's change of food self-sufficiency.

With the continuous improvement of living standards in China, the dietary structure of residents is also changing, and their consumption intentions have shifted from "eating enough" to "eating well," "eating safely," and "eating scientifically." Food security in China faces new challenges (Yang, 2015; Fang et al., 2018) as demand increases for food that is more nutritious, diversified, and healthy. It was also pointed out in the report of the 19th Party Congress that the main contradiction in Chinese society has been transformed into a contradiction between the people's growing demand for a better life and unbalanced and insufficient development. At the same time, numerous studies have shown that the main contradiction in relation to China's food security has shifted from an aggregate shortage to a structural contradiction, highlighted by the coexistence of phased oversupply and undersupply, with the main element of the contradiction being on the supply side (Cui et al., 2018). In terms of high-quality agricultural products with strong market demand and marketability, domestic production and supply are significantly lacking, which leads to a significant increase in imports of medium- and high-end agricultural products. In contrast, despite sufficient domestic production and supply, some large varieties of road goods cannot sell at a price and even a backlog of stagnant sales phenomenon. (IFPRI, 2018). In addition, China's agricultural production costs have continued to rise in recent years, and thus the cost advantage of domestic agriculture has been significantly reduced, the prices of major agricultural products at home and abroad have been "inverted," agricultural imports have increased sharply, some agricultural products have become "foreign goods in the market, national goods in the warehouse," and the lack of competitiveness of domestic agriculture has become increasingly apparent. Thus, although scholars generally agree that China's food security is not a significant problem in the short term, China must remain vigilant regarding the mismatch between supply and demand of livestock products in the medium term and the stability of the supply of bulk agricultural products in the long term (Fan et al., 2021). The core cause of these problems is the mismatch between the agricultural supply structure and the dietary structure of the population; the agricultural products supplied by the domestic market cannot meet the demand of the population, which leads to a considerable reliance on imports of some agricultural products, and declining food self-sufficiency, which threatens national food security.

In the Medium- and Long-term National Food Security Plan (2008-2020), it was clearly stated that "China's food self-sufficiency rate should be maintained at more than 95\%," and the Central Rural Work Conference held in December 2013, which included comprehensive consideration of domestic resources and environmental conditions, food supply and demand patterns, and changes in the international trade environment, established the principle of "We are the first to take the lead." In December 2013, the Central Rural Work Conference established a new national food security strategy of "focusing on us, based on the domestic market, ensuring production capacity, moderate imports and scientific and technological support" and a new food security goal of "ensuring basic grain selfsufficiency and absolute food security." In the new era of socialism with Chinese characteristics, with the changes in the international situation and the transformation of the leading social contradictions, the standard of food-related statistics and the setting of the food self-sufficiency rate have become the focal points of scholars' discussions. The establishment of a scientific and reasonable food self-sufficiency rate target is not only the basis for food security level assessment and food policy 
formulation, but also directly affects the strategic direction and implementation path of the Chinese food industry's development.

Against a background of increasing uncertainty as a result of volatility in the global food supply chain brought about by the novel coronavirus pandemic and the counter-globalization trend, which has hampered international trade, foreign investment, and population and knowledge flows, the formation of a solid domestic market has become the main endogenous driver of China's economic growth and a critical bargaining chip in the international game (Fraser, Legwegoh, \& Krishna, 2015). The Central Economic Work Conference in 2020 proposed "demand-side management," that is, fully exploiting the potential of the domestic market, expanding consumption and effective investment to improve people's livelihoods, and forming a strong domestic market by fostering endogenous power, which provides essential support for building a new development pattern and a solid guarantee for food security. Based on this, it is important to clarify the characteristics of the evolving dietary structure of the population, identify the mechanism of its effect on the food self-sufficiency rate, simulate the level of China's food security in the medium- and long-term future under different scenarios, and refine food security guarantee strategies, as well as to deepen agricultural supply-side reform and achieve a higher-level dynamic balance between food supply and demand in which demand pulls supply and supply creates demand.

\section{LITERATURE REVIEW}

In assessing the food self-sufficiency rate, first it is necessary to clarify the three basic concepts of grain, cereals, and rations. In the narrow sense, "grain" refers to rice, wheat, corn, sorghum, and other grain crops. However, in China, the term "grain" is generally used in the broad sense to include cereals, legumes, and yams, while the term "rations" refers to rice and wheat (Wang et al., 2017). The grain self-sufficiency rate refers to the ratio of grain production to grain consumption. Without considering fluctuations in grain reserves, grain consumption can be regarded as the sum of grain production and net grain imports. Previous studies have generally agreed that the food self-sufficiency rate is directly proportional to the level of food security, that is, the higher the food self-sufficiency rate, the higher the level of food security (Niu et al., 2021). However, there has been debate over the caliber of food-related statistics, and variations in the quality of statistics used in studies have led to conflicting measures of the food self-sufficiency rate. What exactly is China's food self-sufficiency rate? What target should be set for the food self-sufficiency rate? These questions have become the focus of academic debate. Thus, this study assesses the level of food security, calculates the selfsufficiency rates for grain, rations, and cereals, determines the gap between the food self-sufficiency rate and food security in China using different statistical measures, and reveals the evolution and stage characteristics of the relationship between food security and the self-sufficiency rates of grain, rations, and cereals during different periods, to provide decision-making support for the formulation of scientific and reasonable food self-sufficiency targets.

Regarding the evolution of the dietary structure of the population, since the reform and opening up, China's economy has developed rapidly and people's income has been increasing. By the mid-1980s, the problem of national sustenance had been solved, and the dietary structure began to transition to the well-off level, mainly manifested by a significant decrease in the quantity of directly consumed rations and a significant increase in the quantity of animal products consumed (Challinor et al., 2018). Since 1978 , the per capita consumption of food rations in China has decreased by $47 \%$, while the consumption of animal products (meat, milk, and eggs) has increased by $160 \%$ (Hu et al., 2019). Currently, the dietary structure of the population is changing from cereal-based to a healthy and nutritionally diverse structure of cereals, meat, fruits, and vegetables, and there is strong consumption demand, especially for meat, a trend that is consistent with food consumption trends in other developing countries (Cui et al., 2018). Related studies also indicate that China's meat consumption will continue to grow in the future, but the consumption structure has changed, with the proportion of beef and lamb consumption 
increasing and the proportion of pork and poultry consumption decreasing. Thus, beef and lamb will be key components in future agricultural markets. Meanwhile, the early 21 st century is a crucial stage in China's economic development and urbanization, and China's GDP per capita should reach that of medium-level developed countries by 2030 . China's urbanization rate was $56.1 \%$ in 2015 , which is near the midpoint of the accelerated urbanization rate of 30\%-70\%, and is expected to reach about $65 \%$ by 2030 . Based on Engel's law and Bennett's law, the rapid development of the economy and increase in the urbanization level suggest that people's income levels will continue to increase and their dietary structure will continue to improve.

As for the mechanism underlying food self-sufficiency, most previous studies have focused on changes in production and distribution. For example, from the perspective of food supply, the effects of climatic factors such as temperature and precipitation and other factors such as agricultural mechanization, rural labor, arable land area, fertilizers, pesticides, and other inputs to agricultural production on food supply have been identified (Springmann et al., 2018). From the perspective of food distribution, the effects of food price fluctuations, the international environment, and other factors related to food importing and exporting have been examined (Challinor et al., 2018). Overall, most studies have focused on the main "rations" such as wheat and rice, while few studies have analyzed the impact on food self-sufficiency of changes on the consumption side. However, the rapid growth of livestock consumption and production has led to a surge in feed grain use, which has become a major component of current food consumption. Numerous studies have pointed out that the core food security issue has shifted from ration security to feed grain security, and the gap between supply and demand will continue to expand, with China's soybean self-sufficiency rate currently just $15 \%$ (Challinor et al., 2018). The blind pursuit of grain production has led to a mismatch between grain supply and demand, tightening resource and environmental constraints, and making it difficult for farmers to increase their income. This is the main reason for the current decline in grain self-sufficiency and consequent threat to food security (Deng \& Gibson, 2020). In addition, in terms of research methods, most previous studies have used vector autoregressive series models (e.g., Structural Vector Autoregression (SVAR), Markov-Switching Vector Autoregressive (MS-VAR), and Bayesian Vector Autoregression (BSVAR)) to explore the dynamic changes in the relationship between various factors and the food self-sufficiency rate or the gap between food supply and demand based on time series data, but this method does not require a high level of underlying economic theory, does not clearly identify the structural relationships among variables, and ignores the interactions among multiple variables. Thus, this study uses a structural equation model to identify the mechanism influencing the grain self-sufficiency rate in China, measures the observed variables in relation to the grain selfsufficiency rate, and systematically analyzes the transmission path and influence of the evolution of the dietary structure of the population on the future medium- and long-term grain, cereals, and rations self-sufficiency rates. Regarding the analysis of grain demand based on the evolution of the dietary structure of the population, most previous studies have used data released by the state, converting the main food consumed by the population into grain based on specific criteria to calculate the grain demand at either the national level or the urban and rural levels. Most of these studies have involved trend analyses based on the growth of China's population and economy, the urbanization level, and historical changes in grain consumption, combined with economic models, but have ignored the influence of the population's vision of a balanced diet on the evolution of the dietary structure in the new era (Stephens et al., 2020). Numerous studies have shown that the availability and accessibility of diverse nutritious crops required for a healthy diet have been neglected, and that agricultural development programs should consider how to translate agricultural output into good nutrition and better health goals (Cui et al., 2018). However, most previous studies have been based on the reality of a tight grain supply and demand balance in China and have tended to predict trends in rations, feed grain, seed grain, industrial grain, and losses in a disaggregated manner while controlling for grain demand. However, they have generally underestimated the growth in demand for feed grain as a result of the evolution of the dietary structure and the increasing importance of livestock products 
such as meat, milk, and eggs in the grain demand structure. Moreover, their failure to consider the constraining effect of income levels on consumption demand is an issue that needs further attention (Lei et al., 2020). Thus, this study constructs an econometric model from the perspective of balanced diets to identify the food consumption mix that maximizes people's nutrition under the incomelevel constraint, generates the threshold value space of per capita food demand in the medium- and long-term future, reveals the changing trends in people's dietary structure, and analyzes the impact of these changes on total food demand in China in the medium- and long-term future. The findings of this study provide decision-making support regarding the formulation of China's food security strategy in the new era.

In terms of a food security control strategy and guarantee scheme, to adapt to the evolution of the dietary structure of the population and overcome the mismatch between grain supply and demand, the state has introduced a series of control instruments, such as "grain subsidies," "a minimum purchase price for grain," "one billion and eight hundred million acres of arable land red line", and "grain to forage." Scholars have also conducted detailed research on the impacts of various regulatory instruments. For example, the mechanism underlying the impact of the grain subsidy policy on China's food security by 2020 was analyzed using the computable general equilibrium model (Huang et al., 2010). However, previous studies have been based on different assumptions and have ignored the influence of trade-related factors, which leads to significant differences in their results (Mukhopadhyay, Thomassin, \& Zhang, 2018). The 13th Five-Year Plan emphasized for the first time that "we should improve the ability to use international and domestic markets and resources in an integrated manner," while the Fifth Plenary Session of the 19th Party Congress proposed that "a new development pattern should be formed in which the domestic cycle is the main body and the domestic and international cycles promote each other." Trade is a critical means of solving China's food demand and food security problems and is necessary if China is to make full use of both international and domestic resources and markets. However, while relying on the international market to relieve the pressure on domestic food supply, China also faces trade disputes and WTO rules constraints (Anderson, $\&$ Strutt, 2014). Since it joined the WTO, China has adopted a series of tariff concessions, mainly in relation to agricultural products (Hopewell, 2019), and since the outbreak of the Sino-US trade conflict, agricultural products, represented by soybeans, have become an important bargaining chip. Both countries' trade activities have been significantly affected by this conflict (Iqbal, Rahman, \& Elimimian, 2019), especially since the start of the Covid-19 pandemic, which has affected globalization and normalization. Trade restriction measures and expanded coverage have intensified the risk related to stable supply in the international food market (IFPRI, 2018). Thus, this study undertakes comprehensive simulations by constructing different scenarios based on integrating the domestic and international markets to clarify the self-sufficiency rates for grain, cereals, and rations in China in 2030, 2040, and 2050 under different scenarios, analyzes the mechanism underlying the impact of national agricultural regulatory instruments on the self-sufficiency rate of grain under the influence of the international environment, and identifies the performance of different regulatory instruments in terms of guaranteeing food security. The findings of this study provide decision-making support for the formulation of appropriate food self-sufficiency targets and food security strategies in the new era.

\section{RESEARCH QUESTIONS}

This study mainly focuses on the dynamic evaluation of food security and the grain self-sufficiency rate in China, the mechanism whereby the dietary structure evolution influences the grain self-sufficiency rate, predictions of medium- and long-term future food demand based on the evolution of the dietary structure, and regulatory strategies and schemes related to food security in response to the evolution of the dietary structure. The following sub-sections provide detailed descriptions of the relative issues. 


\section{Evaluation Of Food Security and The Grain Self-Sufficiency Rate}

This involves the dynamic evaluation of the restriction mechanism regarding China's food security. Based on the new connotation of food security, this study constructs an index to measure China's food security in the new era, clarifies the level of food security, identifies the principal risks and constraints affecting food security, and reveals the underlying mechanism.

It also involves the dynamic evaluation of the grain self-sufficiency rate and research on the food security gap in China. Consumption statistics are used to measure the self-sufficiency rates of grain, cereals, and rations, and to determine the grain self-sufficiency rate and food security gap under the different statistical standard. This assessment explores the evolutionary history and stage characteristics of the relationship between food security and food, cereal, and ration self-sufficiency over time.

\section{Forecasts Of Medium- And Long-Term Future Food Demand}

This involves analysis of the medium- and long-term future trends in dietary structure. Based on the "Dietary Guidelines for Chinese Residents (2016)" and the current dietary structure, the per capita food demand and its components under the balanced diet mode and the current development mode were calculated. Key parameters such as population, GDP, urbanization rate, income elasticity, and agricultural product prices were calibrated to identify future trends in the dietary structure and the driving mechanism.

It also involves forecasting medium- and long-term future grain demand based on the evolution of the dietary structure. Based on the threshold value space of medium- and long-term future per capita grain demand, total medium- and long-term future grain demand was calculated using the "grain consumption conversion coefficient." With the help of the "feed grain conversion rate," the amounts of various kinds of grain (e.g., corn, wheat, and rice) necessary to meet the per capita food demand were identified, which provides crucial information for the formulation of appropriate food security strategies and schemes.

\section{Food Security Strategies And Schemes Based On Evaluating The Dietary Structure}

The international environment and national agricultural control measures were included in the analysis framework. Combined with the mechanism whereby the evolution of the residents' dietary structure affected the grain self-sufficiency rate and forecasts for medium- and long-term future grain demand, parameters were set regarding various aspects of production, consumption, and international trade to simulate the changing grain, cereals, and rations self-sufficiency rates and the food security gaps under different scenarios.

Based on the results of the parameter analysis, this study forecasts the self-sufficiency rates for grain, cereals, and rations in China in 2030, 2040, and 2050, and systematically depicts the underlying mechanism for "one billion and eight hundred million acres of arable land red line," "grain to forage," and other agricultural controls relating to food security. This is combined with various food security strategies to refine China's food security control strategies and security programs in the new era.

\section{Key Scientific Questions Regarding China's Food Self-Sufficiency}

Against a background of fluctuations in the global food supply chain and increasing uncertainty caused by the trend toward anti-globalization, it is vital to clarify the evolution of Chinese residents' dietary structure and identify the mechanism influencing its effect on the food self-sufficiency rate. By analyzing the level of China's food security and its food self-sufficiency rate in different periods, this study summarizes the current risks in relation to China's food security, identifies the mechanism whereby the evolution of the dietary structure has influenced China's food self-sufficiency rate since the reform and opening up, and refines the food security strategies necessary to adapt to the evolution of the dietary structure. 


\section{APPROACHES AND METHODS}

\section{The Evolution Of The Dietary Structure And Its Effect On The Grain Self-Sufficiency Rate}

Based on historical consumption data, from the perspective of "grain in the broad sense, "the per capita consumption of grain, meat, milk, eggs, seafood, fruits, vegetables, and other foods is selected as a key index, and the evolution of the various types of food consumption since the reform and opening up is systematically examined to identify differences in the dietary structures of urban and rural residents. First, the "grain consumption conversion coefficient" of different agricultural products is defined, and the proportion of various types of foods (e.g., grain, meat, eggs, and milk) in the residents' dietary structure in different periods is calculated to provide parameters for analyzing the mechanism whereby the evolution of the dietary structure influences the food self-sufficiency rate. Second, combining qualitative analysis with quantitative analysis, we explore the transmission path and influence mechanism of the evolution of the residents' dietary structure on the grain self-sufficiency rate and qualitatively analyze and refine the theoretical basis, research focus, and development trend of the influence mechanism of the grain self-sufficiency rate in China. Finally, the factors influencing the grain self-sufficiency rate in China are calculated, the contribution rate and effect intensity are revealed, and the mechanism of influence of the evolution of the dietary structure of residents is quantitatively identified.

\section{Prediction And Evaluation Of The Grain Self-Sufficiency Rate In China}

In this study, consumption statistics are used to calculate direct consumption, feed consumption, industrial consumption, seed reserves, and losses. Combined with the feeding standards for cattle, sheep, pigs, chickens, and other livestock and poultry, and the "feed grain conversion rate," the quantities of various kinds of grain (e.g., corn, wheat, and rice) in the residents' dietary structure are identified. Using total grain production data, the self-sufficiency rates for grain, cereals, and rations in China from 2000 to 2020 were calculated to identify the self-sufficiency rate and food security gap in relation to China's grain production under different statistical dimension. By changing key nodes, the evolution course, and the stage characteristics of the relationship between food security and grain, the self-sufficiency rates of grain and rations in different periods were identified. The evolution course and stage characteristics of the relationship between food security and the selfsufficiency rates for grain, cereals, and rations in various future periods could then be inferred. The results provide support for decision-making regarding the formulation of scientific and reasonable grain self-sufficiency rate targets and for forming a "higher level of the dynamic balance between demand and supply to pull the supply side ".

\section{Suggestions And Countermeasures Regarding The Grain Self-Sufficiency Rate}

This involves identifying medium- and long-term future trends in the dietary structure of residents, analyzing the driving mechanism, identifying the per capita demand for rations, feed grain, industrial grain, and seed grain, estimating total grain demand in the medium- and long-term future, and identifying the impact of the evolution of residents' dietary structure on total grain demand.

Against a background of a declining grain self-sufficiency rate and continuous improvement in the residents' dietary structure (Figure 1), this study examines the influence of dietary structure improvement on the grain self-sufficiency rate and puts forward countermeasures to ensure that the grain self-sufficiency rate guarantees China's food security in relation to import and export strategies, holding back the weakening of agricultural production, ensuring self-sufficiency in the three main types of grain, classifying and determining the food self-sufficiency rate, and proposing the policy system to ensure food security. 
Figure 1. Framework of dietary structure change and its impact on the grain self-sufficiency rate

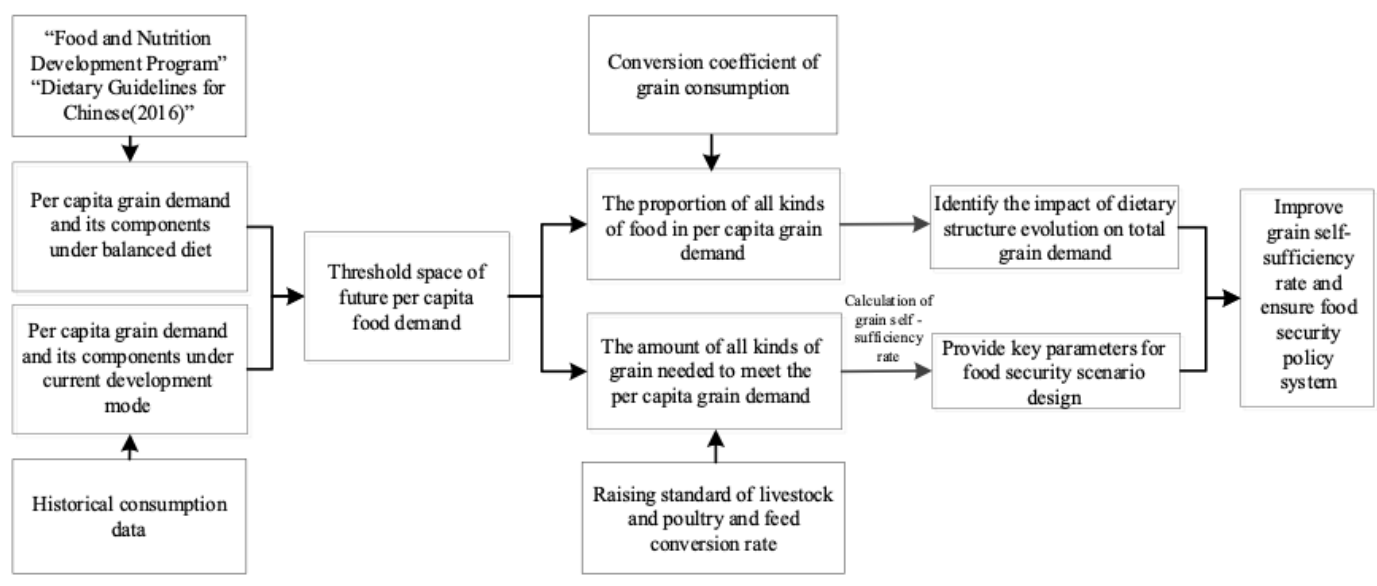

\section{Calculation Of the Grain Self-Sufficiency Rate}

The grain self-sufficiency rate is the ratio of total grain production to total grain consumption, and can be expressed as follows:

$$
w=G / D \times 100 \%,
$$

where $\mathrm{w}$ is the grain self-sufficiency rate, $\mathrm{G}$ is total grain production, and $\mathrm{D}$ is total grain consumption.

The key to measuring the grain self-sufficiency rate is determining the grain statistical dimension and total grain consumption. Based on the research goals, this study uses the consumption-based method for specific accounting. Generally speaking, grain consumption is divided into five categories: direct consumption, feed consumption, industrial consumption, seed reservation, and losses during transportation and storage. Direct consumption refers to grain directly consumed by residents. Feed consumption refers to grain consumed in the production of animal-based food products such as meat, eggs, and milk. The feeding standards for cattle, sheep, pigs, chickens and other poultry, and other livestock issued by the Ministry of Agriculture were obtained, and then the indirect consumption of various types of grain was calculated by applying the feed grain conversion rates. Xu et al. (2014) and Zhan (2019) found that the feed grain conversion rate is $4.1: 1$ for beef and mutton, 4.6:1 for pork, 4.6:1 for animal oil, 3.2:1 for poultry meat, 3.6:1 for eggs, 2.0:1 for seafood, 0.8:1 for fresh milk, and $72: 1$ for alcohol. Industrial consumption refers to grain consumed in the production of raw materials or auxiliary materials, such as those used in food processing and starch production. Industrial grain consumption statistics reveal that the average annual growth rate of China's total industrial food consumption is $6.8 \%$, and rice, wheat, corn, potato, and soybean account for $14.04 \%, 15.23 \%, 45.79 \%$, $7.40 \%$, and $14.40 \%$, respectively. Based on these figures, the annual industrial consumption of the corresponding food types was calculated. In addition, there were losses in relation to grain sowing, harvesting, transportation, storage, processing, and consumption. To ensure normal grain reproduction, a certain amount of grain should be reserved as seed grain. The amount of grain reserved, including losses, is based on the findings of Yang et al. (2019), that is, 5\% of the total annual output. Thus, summing the five types of grain consumption, total grain consumption is calculated as follows: 


$$
\begin{aligned}
& G_{d}=P_{u} \times d_{u}+P_{r} \times d_{r} \\
& G_{c}=P_{u} \times \sum_{i=1}^{9} U_{i} \times a_{i}+P_{r} \times \sum_{i=1}^{9} R_{i} \times a_{i}, \\
& D=G_{d}+G_{c}+G_{i n}+G_{\text {seed }}+G_{\text {loss }}
\end{aligned}
$$

where D represents total grain consumption, Gd represents direct consumption, $G_{c}$ represents feed grain consumption, $G_{i n}$ represents industrial grain consumption, $G_{\text {seed }}$ represents seed grain consumption, and $G_{\text {loss }}$ represents losses. $P_{u}$ and $P_{r}$ represent the total population in urban and rural areas, respectively, $d_{u}$ and $d_{r}$ represent per capita direct annual consumption by urban and rural residents, respectively, and $U_{i}$ and $R_{i}$ represent per capita consumption by urban and rural residents, respectively, of various foods where $i$ is a number from 1 to 9 such that $1=$ pork, $2=$ beef, $3=$ lamb, $4=$ animal oil, $5=$ seafood, $6=$ poultry meat, $7=$ eggs, $8=$ fresh milk, and $9=$ wine. $a$ is the conversion rate for feed and grain.

\section{Conversion Coefficient of Grain Consumption}

Using historical consumption data combined with the results of the PMP model, this study transformed all types of food (e.g., rations, meat, eggs, and milk) from the per capita grain demand into "raw food" and calculated total grain demand and the proportion of all types of foods in the residents' dietary structure in the medium- and long-term future. In recent years, animal husbandry in China has shifted from bulk breeding to large-scale breeding. Therefore, the conversion coefficient of consumption for pork, chicken, eggs, and milk used in this study is based on large-scale breeding data. Large-scale breeding data for beef and mutton were unavailable, and thus bulk-breeding data were used. The grain consumption conversion coefficient formula for pork, beef, and mutton is: grain consumption conversion coefficient $=$ grain consumption quantity/individual meat output. The calculations for eggs, milk, and fish do not include the problem of meat yield, and so the ratio of the quantity of grain consumed to the output of the main product is used to calculate the grain consumption conversion coefficient. The carcass rate for chicken is the industry standard of $71.8 \%$, and thus the food consumption conversion coefficient for chicken is: quantity of food consumed/ (yield of main product $\times$ carcass rate). The liquor coefficient is calculated based on the conversion standard of 1:3, while the beer coefficient is calculated based on the conversion standard of 1:0.2. Because the residue from vegetable oil extraction mainly enters the animal husbandry industry as feed, to avoid double calculation, this study uses a grain conversion coefficient for edible oil consumption of 1 . Milk powder is converted into fresh milk at a ratio of 1:10. As the rations of urban residents are processed grain data, this study uses the grain yield of 0.75 to convert rations back to raw grain (see Table 1).

\section{RESULTS}

\section{Changes In The Dietary Pattern}

In 1949, annual per capita food consumption in China was only $209 \mathrm{~kg}$. However, the great economic and technological progress that has occurred in recent decades has seen this figure increase significantly, to the point where current annual per capita food output is around $470 \mathrm{~kg}$, which is above the global average, and means that 1.4 billion people in China are now completely free from hunger. Despite having only $5 \%$ of the world's freshwater resources and $8 \%$ of its arable land resources, China is able to support nearly $20 \%$ of the world's population (see Figure 2).

The dietary pattern of residents has undergone significant changes since the 1950s and 1960s, and rations consumption as a proportion of overall food consumption has decreased significantly (see Table 1). In addition, annual per capita grain consumption has increased from $350.8 \mathrm{~kg}$ to 457.6 $\mathrm{kg}$, an increase of $30.5 \%$, rations consumption has increased from $211.6 \mathrm{~kg}$ to $247 \mathrm{~kg}$, an increase 
Table 1. Conversion coefficients for the main food types consumed in China

\begin{tabular}{|l|l|l|l|l|l|l|l|}
\hline & Pork & Beef & Lamb & Chicken & Eggs & Milk & Fish \\
\hline Grain consumption/kg & 217.3 & 367.1 & 45.7 & 398.6 & 2957.7 & 2207.5 & 724.3 \\
\hline Output of main products /kg & 114.9 & 418.0 & 43.0 & 231.6 & 1752.6 & 5572.6 & 737.1 \\
\hline Individual meat production /(kg/pcs) & 75.9 & 140.7 & 15.0 & 71.8 & & & \\
\hline $\begin{array}{l}\text { Conversion coefficient of grain } \\
\text { consumption/(kg/kg) }\end{array}$ & 2.9 & 2.6 & 3.1 & 2.4 & 1.7 & 0.4 & 1.0 \\
\hline
\end{tabular}

Note: The chicken carcass percentage is the industry standard of $71.8 \%$.

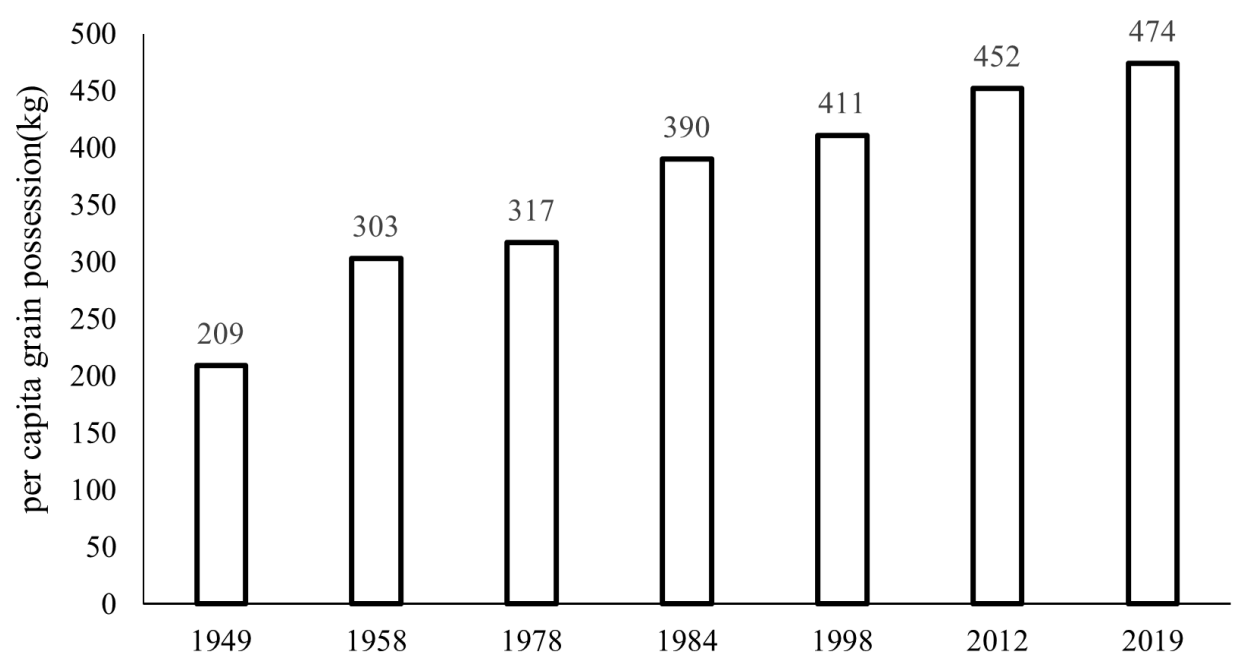

of $9.91 \%$, meat consumption has increased from $49.1 \mathrm{~kg}$ to $63.7 \mathrm{~kg}$, an increase of $29.8 \%$, vegetable consumption has increased from $377.3 \mathrm{~kg}$ to $550.3 \mathrm{~kg}$, an increase of $45.8 \%$, fruit consumption has increased from $51.7 \mathrm{~kg}$ to $122 \mathrm{~kg}$, an increase of $136 \%$, sugar consumption has increased from 7.7 $\mathrm{kg}$ to $12.4 \mathrm{~kg}$, an increase of $60.8 \%$, and vegetable oil consumption has increased from $10.7 \mathrm{~kg}$ to $22.9 \mathrm{~kg}$, an increase of $112.7 \%$ (Table 2).

The consumption of livestock products has increased significantly. However, per capita consumption of livestock products (e.g., meat, milk, and eggs) continues to account for a lower proportion of total food consumption than that in developed countries in Europe and in the United States, and the proportion of dairy products consumed continues to be below the global average (see Figure 3). Meat consumption was dominated by pork and poultry, with beef and mutton accounting for a relatively low proportion. The consumption of livestock products will continue to increase significantly in the future.

China's agricultural industry has failed to make timely adjustments in response to increasing demand, and the industrial structure and backward modes of production have led to a serious shortage of high-quality forage. This has made it difficult to meet the demand for livestock products, and 
Table 2. Changes in annual per capita consumption of major food types in China (kg)

\begin{tabular}{|l|l|l|l|}
\hline Foods & $\mathbf{2 0 0 1}$ & $\mathbf{2 0 1 0}$ & $\mathbf{2 0 1 5}$ \\
\hline Meat & 49.1 & 57.9 & 63.7 \\
\hline Poultry and Eggs & 17.5 & 20.4 & 21.0 \\
\hline Milk & 8.4 & 29.1 & 33.7 \\
\hline Grain & 350.8 & 411.1 & 457.6 \\
\hline Domestic soybeans & 12.1 & 11.2 & 8.9 \\
\hline Vegetables & 377.3 & 478.9 & 550.3 \\
\hline Fruits & 51.7 & 95.5 & 122.0 \\
\hline Aquatic products & 34.6 & 40.4 & 47.3 \\
\hline Sugar & 7.7 & 10.3 & 12.4 \\
\hline Vegetable oil & 10.7 & 19.7 & 22.9 \\
\hline
\end{tabular}

resulted in an overdependence on imports. The overall trends in beef, lamb, milk powder, and liquid milk imports are rising and increasing in volatility (see Figure 4).

Especially in the context of trade friction between China and the United States, improving selfsufficiency in relation to livestock products is of great significance in relation to China's food security. In 2018, rations, industrial grain, feed grain, seed grain, and other grain accounted for $28.5 \%, 17.4 \%$, $41.5 \%, 1.5 \%$, and $11.1 \%$, respectively, of total demand for grain in China. The proportion of rations have been changed over years, while that of industrial and feed grain increased and that of seed and other grain remained relatively stable (see Figure 5).

Figure 3. Per capita consumption of meat, milk, and eggs as a proportion of total per capita food consumption in 2015

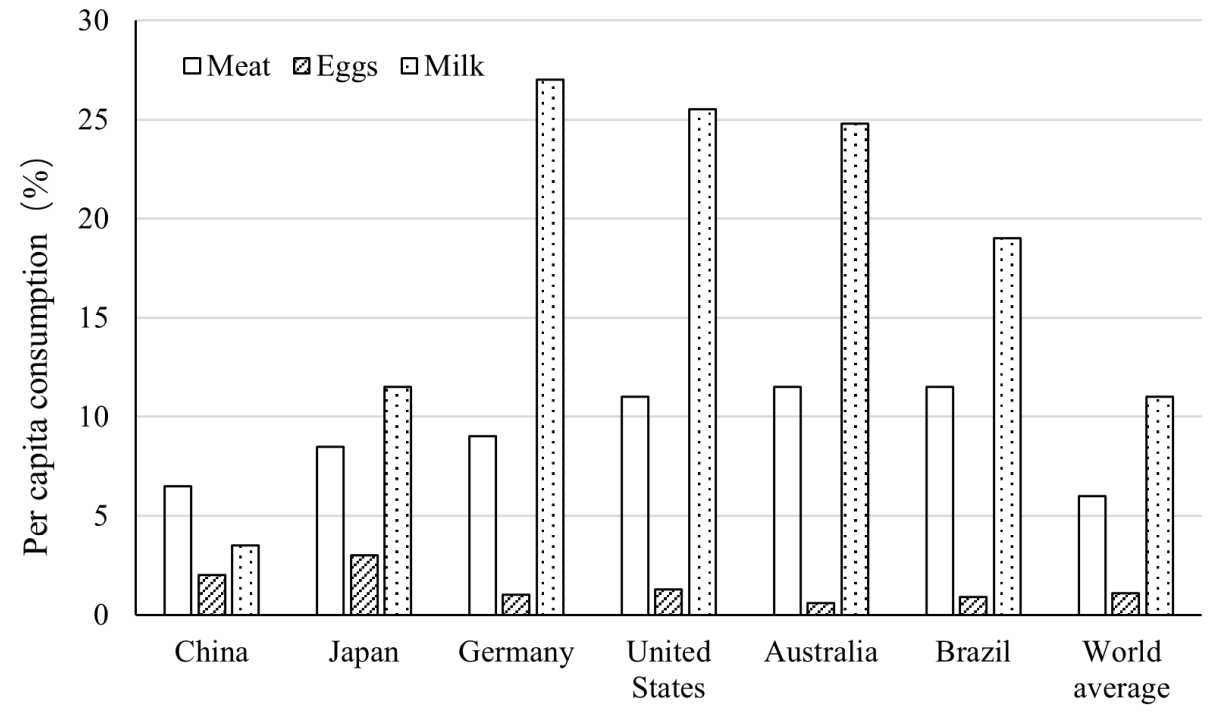




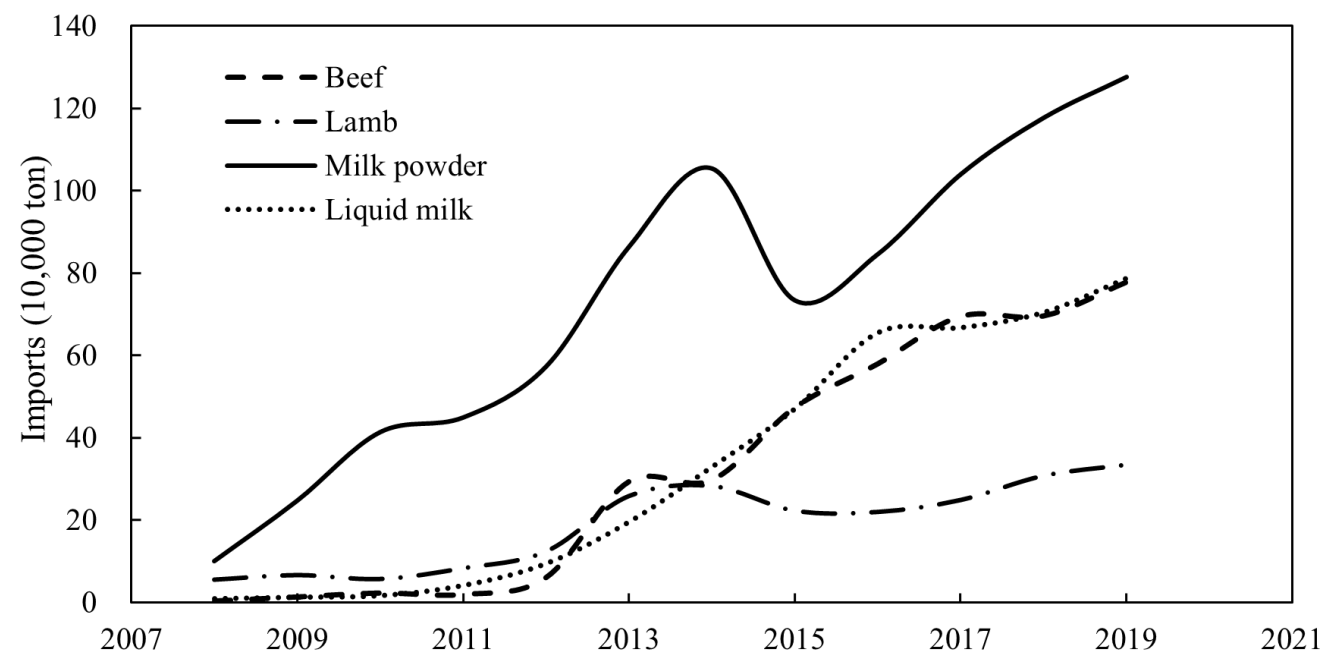

Figure 5. Grain demand in China, 2007-2020

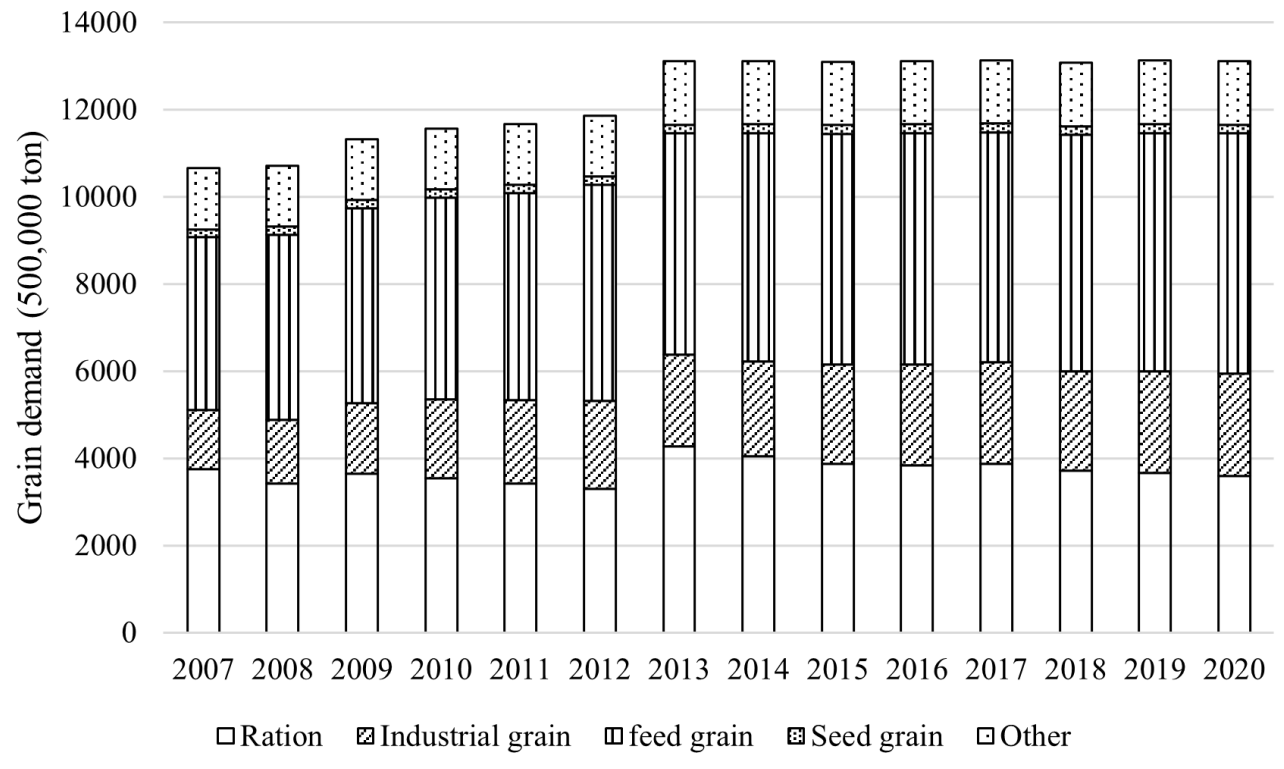

\section{Changes In The Food Self-Sufficiency Rate}

China's rapid economic and social development and improved living standards have seen the dietary structure of residents become more diversified, and thus consumption of feed and industrial grain has increased. The demand for food has also increased significantly, resulting in increased food imports. 


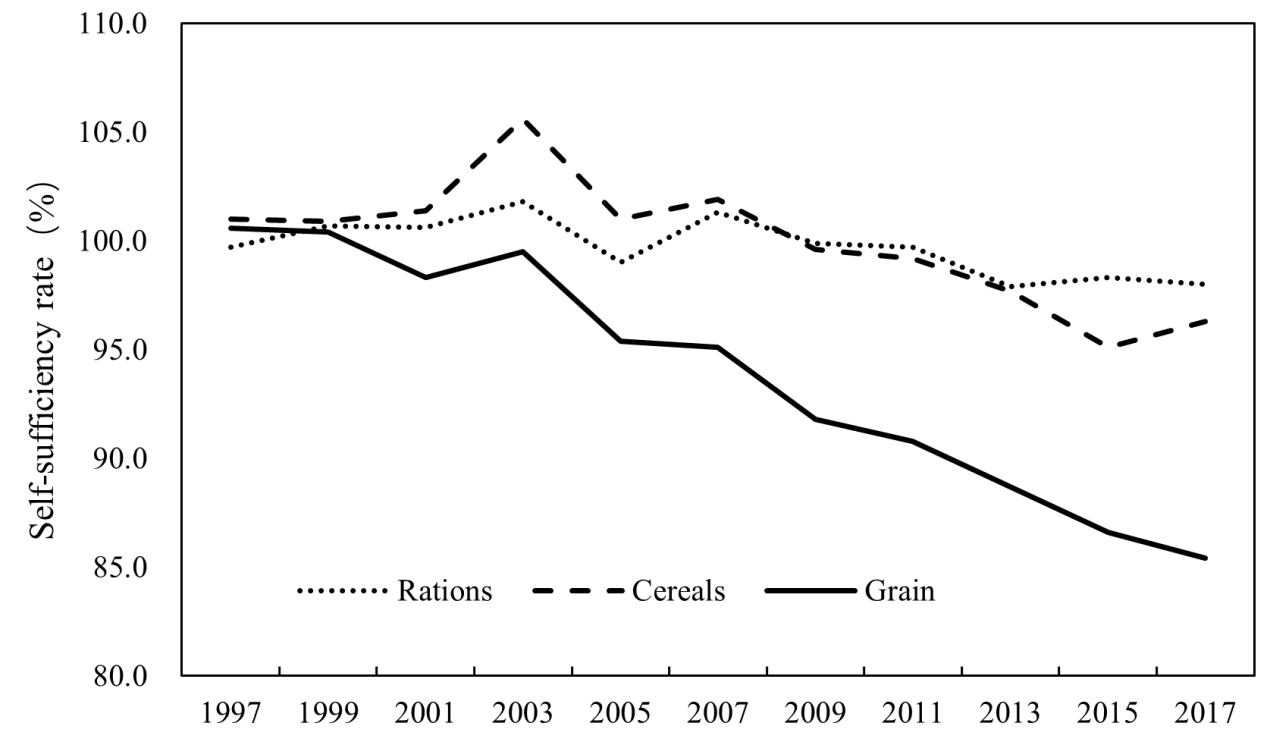

Current annual per capita food consumption is far in excess of $400 \mathrm{~kg}$, and the food self-sufficiency rate has begun to show a downward trend. From 1997 to 2017, the food self-sufficiency rate was in continuous decline (see Figure 6). In 1997, the grain self-sufficiency rate was $100.6 \%$, but it fell to 98.3\% in 2001 before increasing to $99.5 \%$ in 2003 , after which it continued to decrease. The cereals self-sufficiency rate was $100.6 \%$, but it fell to $95.1 \%$ in 2015 before increasing to $96.3 \%$ in 2017 . With the support of national policies, grain production in China has continuously increased, but the substantial increase in demand, driven by the surge in population and increased demand for feed grain and industrial grain has seen the grain self-sufficiency rate continue to decline.

\section{The Effect Of Changes In The Dietary Pattern On The Food Self-Sufficiency Rate}

The dietary pattern has an impact on the food self-sufficiency rate in terms of both production and consumption. From the perspective of consumption, the residents' choice of plant-based food products and animal-based food products was reflected in their choice of rations and feed grain, respectively. Rations and feed grain were the most important requirements for food production, therefore the dietary pattern of residents played a decisive role in the demand for food. Improvements in the dietary pattern caused the residents' plant-based food product consumption to continue to decrease, while animal-based food product consumption continued to increase. The production of animal-based food products would consume more food. The upgrading of the dietary pattern could have an impact on total food consumption. From the perspective of production, changes in the dietary pattern have led to an increasing share of non-cereal agricultural products, such as livestock products and fruits and vegetables, in grain production, resulting in a decline in the competitiveness of grain production in terms of labor, water, and land resources, and supporting policies, thereby reducing the ability to increase grain production.

Grain consumption and production are important factors affecting the food self-sufficiency rate, and thus changes in the dietary pattern have a significant impact on the food self-sufficiency rate. An increase in per capita income increases the purchasing power of residents, which leads to an increase in overall food consumption; this has an impact on the food self-sufficiency rate. An increasing 
population and increased overall food consumption has made it difficult to ensure China's food selfsufficiency. Cultivated land is the basis of food production. If a country can maintain sufficient arable land, it can maintain its production capacity, thereby ensuring food self-sufficiency.

Table 4. Production, consumption, and forecast self-sufficiency rates for rice, wheat, corn, beans, and all grain

\begin{tabular}{|c|c|c|c|c|c|}
\hline Food & Indicators & 2020 & 2030 & 2040 & 2050 \\
\hline \multirow{3}{*}{ Rice } & production & 21792.4 & 25290.9 & 27716.4 & 30374.5 \\
\hline & consumption & 20233.0 & 17801.5 & 15827.1 & 14475.5 \\
\hline & self-sufficiency rate (\%) & 107.7 & 142.07 & 175.12 & 209.83 \\
\hline \multirow{3}{*}{ Wheat } & production & 13545.5 & 15413.1 & 16857.8 & 18438.0 \\
\hline & consumption & 12454.2 & 11219.6 & 10283.3 & 9748.5 \\
\hline & self-sufficiency rate (\%) & 108.7 & 137.38 & 163.93 & 189.14 \\
\hline \multirow{3}{*}{ Corn } & production & 26832.8 & 32073.3 & 35428.9 & 39135.5 \\
\hline & consumption & 30292.6 & 40415.7 & 52949.9 & 68474.4 \\
\hline & self-sufficiency rate $(\%)$ & 88.58 & 79.36 & 66.91 & 57.15 \\
\hline \multirow{3}{*}{ Beans } & production & 1773.5 & 2204.6 & 2558.5 & 2969.3 \\
\hline & consumption & 10159.6 & 8500.1 & 7004.0 & 5740.1 \\
\hline & self-sufficiency rate (\%) & 17.46 & 25.94 & 36.53 & 51.73 \\
\hline \multirow{3}{*}{ Grain } & production & 63944.1 & 74981.9 & 82561.6 & 90917.3 \\
\hline & consumption & 75121.4 & 80571.9 & 89509.8 & 102889.6 \\
\hline & self-sufficiency rate $(\%)$ & 85.12 & 93.06 & 92.24 & 88.36 \\
\hline
\end{tabular}

Note: measured in units of 10,000 tonnes.

\section{Food Security Control And Guarantee Strategies In Response To Changes In Residents' Dietary Patterns}

It can be seen from Table 4 that China's self-sufficiency rate for rice, wheat, and beans is expected to increase continuously from $107.71 \%, 108.76 \%$, and $17.46 \%$, respectively, in 2020 to $209.83 \%$, $189.14 \%$, and $51.73 \%$, respectively, in 2050 . However, the self-sufficiency rate for corn is expected to decrease continuously from $88.58 \%$ in 2020 to $57.15 \%$ in 2050 . Therefore, the overall food selfsufficiency rate is expected to rise from $85.12 \%$ in 2020 to $93.06 \%$ in 2030 , and then gradually decrease to $88.36 \%$ in 2050 (Figure 7). 


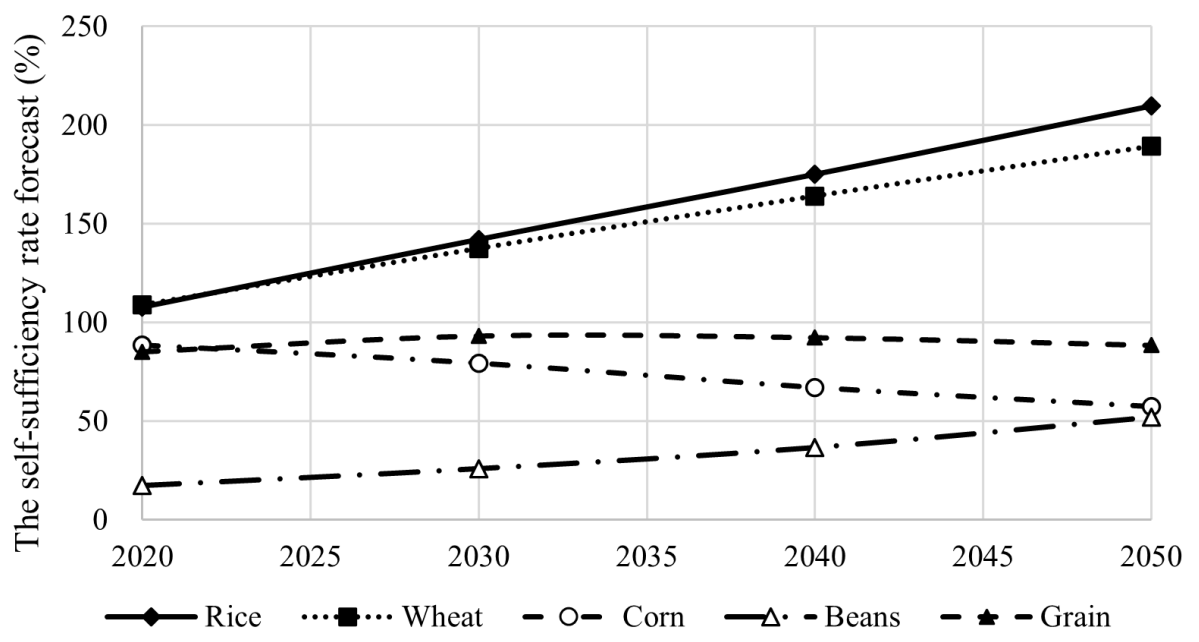

Note: (Interpolated using linear trends, derived from population, GDP, and urbanization rate)

The results of this study showed that per capita consumption of plant-based food products such as rice and wheat has declined. However, consumption of corn and soybeans has increased because of an increase in demand for feed grain and processing grain. Moreover, per capita consumption of animalbased food products such as meat, milk, and seafood has increased. Based on the food consumption forecasts from 2014 to 2030, domestic plant-based food product consumption has continued to decrease, while animal-based food product consumption has continued to increase. Therefore, with the ongoing development of China's economy, the future dietary structure is likely to shift further toward animal-based food products, and thus consumption of meat, milk, and seafood will continue to increase. Based on a review of the historical evolution of the food self-sufficiency rates in China and other countries, the following policy recommendations are proposed.

First, overall grain production capacity should be increased. Given China's large population and limited water and arable land resources, domestic food production is the foundation of social harmony and national stability. To meet the demands of the domestic market, it is necessary to increase overall grain productivity. Water and soil are the most important primary resources for agricultural production. Thus, China must further improve its laws and regulations to reduce phenomena such as arbitrary wastage of cultivated land, land pollution, and wastage of water resources. In addition, China should promote innovation in relation to agricultural science and technology, and the reform of the agricultural scientific research and extension system, improve the productivity of low- and medium-yield arable land, and fully pursue the maximum potential grain yields. Finally, it is necessary to improve agricultural infrastructure, increase investment in basic production facilities such as farmland water conservation, improve agricultural production conditions for ensuring food security.

Second, the grain importing strategy should be adjusted to protect food rations moderate liberalization of feed grain. A general emphasis on a higher food self-sufficiency rate is neither realistic nor in line with economic principles. Rather, it is necessary to clarify the strategic focus of food security guarantees, import food in moderation, and rationally allocate precious domestic agricultural production resources. China should increase its support for rice, wheat, and other rations related to fundamental food security to ensure that it has absolute ration security. However, corn showed a net import result for the first time in 2010, and increasing consumption of animal-based food products 
in China means that increasing corn imports are inevitable. To alleviate the shortages of water and arable land, China can moderately liberalize imports of grain other than rations, expand its use of the international market, make good use of global food resources, and use domestic agricultural resources for the production of rations to ensure security of supply.

Finally, knowledge regarding nutrition should be made widely available to scientifically guide residents' consumption patterns. At present, plant-based food, products dominate the dietary structure of residents; however, the consumption of cereals is decreasing compared of animal-based food products such as meat, eggs, milk, and seafood. Given China's rapid economic development and the continuous increase in residents' incomes, the dietary structure will gradually develop towards the trend of plant and animal food being as important in the future.

\section{CONCLUSION AND DISCUSSION}

\section{Major Conclusions}

This study explores changes in China's food self-sufficiency rate in the context of a changing dietary structure over different periods. At the same time, an examination of China's dietary structure and food self-sufficiency rate reveals that China feeds nearly $20 \%$ of the world's population despite having only $5 \%$ of the world's freshwater resources and $8 \%$ of the world's arable land resources. The dietary structure of China's population has changed significantly since the 1950s and 1960s. The consumption of rations as a proportion of overall food consumption has fallen significantly. In addition, the supply of high-quality forage is insufficient, making it difficult to meet the demand for livestock products, resulting in a reliance on imports for many of these products. Hence, China's level of dependence on foreign food producers is increasing.

Focusing on decision-making in relation to major national strategies, and based on the reality of "the imbalance between food supply and demand caused by the evolution of the dietary structure of the population," we explore the impact of the evolution of the dietary structure of the population on national food security using the food self-sufficiency rate under various statistical dimensions as the observed variable, and simulate various food security scenarios for China in the medium- and longterm future. As a result, a food security guarantee strategy is identified. It was found that China's rapid economic and social development and the continuous improvement in people's living standards has led to a decline in the food self-sufficiency rate. Analysis of data regarding the factors influencing the food self-sufficiency rate revealed that the improved dietary structure, involving an increase in animal-based food product consumption, has led to a decrease in China's food self-sufficiency rate. Furthermore, the dietary structure is likely to continue to move toward increased consumption of animal-based food products in the future. Therefore, it is likely that China's grain self-sufficiency rate will continue to decline. The results of this study will assist decision-makers in formulating scientific and reasonable food self-sufficiency rate targets and food security strategies.

\section{DISCUSSION}

Based on "the imbalance of food supply and demand caused by the evolution of the residents' dietary structure," relevant experts are invited to guide and demonstrate the research plan and organize the research content according to the idea of "variable and parameter preparation-influence mechanism discussion-guarantee strategy refinement." Based on data regarding the food security level and food self-sufficiency rates in different periods, the characteristics of the evolution of the residents' dietary structure were identified. Using the level of food security and the food self-sufficiency rate under different statistical dimensions as observational variables, this study explored the impact of the evolution of residents' dietary structure on national food security and developed appropriate food security assurance strategies. However, many factors affect the food self-sufficiency rate, including 
the price of food. The grain price is a complex issue involving a range of factors including the spot market, futures market, supply, demand, trade, the domestic market, and foreign markets. Therefore, the timely update of price-related data is a critical issue that should be further analyzed.

The selection method used in this study inevitably involved subjectivity regarding the selection of independent variables, and selection of different variables would obviously produce different results. Furthermore, the design of different simulation scenarios combining reality also need to be further studied in the future. Nevertheless, this study is focused on one of China's significant strategic challenges. Therefore, it combines the research paradigms of resource management and policy science in attempting to generate decision-making information that will aid the formulation of appropriate national strategies, focusing on the integrity of the research system while striving to achieve clear and specific objectives, and providing a complete description of the critical technology approaches and implementation paths.

\section{ACKNOWLEDGMENT AND FUNDING AGENCY}

This research was funded by the Strategic Priority Research Program of Chinese Academy of Sciences [grant number XDA23070400] and the National Natural Science Foundation of China [grant number 41771568; grant number 72042020]. 


\section{REFERENCES}

Anderson, K., \& Strutt, A. (2014). Food security policy options for China: Lessons from other countries. Food Policy, 49, 50-58. doi:10.1016/j.foodpol.2014.06.008

Challinor, A. J., Neil, A. W., \& Benton, T. G. (2018). Transmission of climate risks across sectors and borders. Philosophical Transactions - Royal Society. Mathematical, Physical, and Engineering Sciences, 376(2121), 20170301. doi:10.1098/rsta.2017.0301 PMID:29712795

Cui, Z. L., Zhang, H. Y., \& Chen, X. P. (2018). Pursuing sustainable productivity with millions of smallholder farmers. Nature, 555(7696), 363-366. .10.1038/nature25785

Deng, X., \& Gibson, J. (2020). Sustainable land use management for improving land eco-efficiency: A case study of Hebei, China. Annals of Operations Research, 290(1), 265-277. doi:10.1007/s10479-018-2874-3

Fan, S., Teng, P., \& Ping, C. (2021). Food system resilience and COVID-19-Lessons from the Asian experience. Global Food Security, 28, 100501. doi:10.1016/j.gfs.2021.100501

Fang, J., Jing, H., Zhang, W., Gao, S., Duan, Z., Wang, H., Zhong, J., Pan, Q., Zhao, K., Bai, W., Li, L., Bai, Y., Jiang, G., Huang, J., \& Huang, Z. (2018). The concept of "Grass-based Livestock Husbandry" and its practice in Hulun Buir, Inner Mongolia. Chinese Science Bulletin, 63(17), 1619-1631. doi:10.1360/N972018-00042

Fraser, E. D. G., Legwegoh, A., \& Krishna, K. C. (2015). Food stocks and grain reserves: Evaluating whether storing food creates resilient food systems. Journal of Environmental Studies and Sciences, 5(3), 445-458. doi:10.1007/s13412-015-0276-2

Hopewell, K. (2019). US-China conflict in global trade governance: The new politics of agricultural subsidies at the WTO. Review of International Political Economy, 26(2), 207-231. doi:10.1080/09692290.2018.1560352

Hu, Z., Zhao, Z., Zhang, Y., Jing, H., Gao, S., \& Fang, J. (2019). Does 'Forage-Livestock Balance' policy impact ecological efficiency of grasslands in China? Journal of Cleaner Production, 207, 343-349. doi:10.1016/j. jclepro.2018.09.158

Huang, D. L. (2010). Impact of agricultural subsidies on Chinese food security based on China agricultural CGE model. Zhongguo Nongxue Tongbao, 26, 429-435.

International Food Policy Research Institute (IFPRI). (2018). 2018 Global food policy report. International Food Policy Research Institute (IFPRI).

Iqbal, B. A., Rahman, N., \& Elimimian, J. (2019). The future of global trade in the presence of the Sino-US trade war. Economic and Political Studies, 7(2), 217-231. doi:10.1080/20954816.2019.1595324

Lei, L. I., Zhai, S. X., \& Bai, J. F. (2020). The dynamic impact of income and income distribution on food consumption among adults in rural China. Journal of Integrative Agriculture, 20(1), 330-342. doi:10.1016/ S2095-3119(20)63239-7

Mukhopadhyay, K., Thomassin, P. J., \& Zhang, J. (2018). Food security in China at 2050: A global CGE exercise. Journal of Economic Structures, 7(1), 1. doi:10.1186/s40008-017-0097-4

Niu, Y., Xie, G., Xiao, Y., Liu, J., Wang, Y., Luo, Q., Zou, H., Gan, S., Qin, K., \& Huang, M. (2021). Spatiotemporal Patterns and Determinants of Grain Self-Sufficiency in China. Foods, 10(4), 747. doi:10.3390/ foods10040747 PMID:33915967

Renard, D., \& Tilman, D. (2019). National food production stabilized by crop diversity. Nature, 571(7764), 257-260. doi:10.1038/s41586-019-1316-y PMID:31217589

Springmann, M., Clark, M., Mason-D’Croz, D., Wiebe, K., Bodirsky, B. L., Lassaletta, L., de Vries, W., Vermeulen, S. J., Herrero, M., Carlson, K. M., Jonell, M., Troell, M., DeClerck, F., Gordon, L. J., Zurayk, R., Scarborough, P., Rayner, M., Loken, B., Fanzo, J., \& Willett, W. et al. (2018). Options for keeping the food system within environmental limits. Nature, 562(7728), 519-525. doi:10.1038/s41586-018-0594-0 PMID:30305731

Stephens, E. C., Martin, G., \& Wijk, M. V. (2020). Editorial: Impacts of COVID-19 on agricultural and food systems worldwide and on progress to the sustainable development goals. Agricultural Systems, 183, 102873. doi:10.1016/j.agsy.2020.102873 PMID:32501358 
Wang, J., Li, Y., Huang, J., Yan, T., \& Sun, T. (2017). Growing water scarcity, food security and government responses in China. Global Food Security, 14, 9-17. doi:10.1016/j.gfs.2017.01.003

Yang, M. (2015). Analysis and prediction of Chinese medium and long term total demand of grain and economic structure. Advance Journal of Food Science and Technology: AJFST, 9(5), 322-325. doi:10.19026/ajfst.9.1909

Zhan, S. (2017). Riding on self-sufficiency: Grain policy and the rise of agrarian capital in China. Journal of Rural Studies, 54, 151-161. doi:10.1016/j.jrurstud.2017.06.012 